\title{
Increased prevalence of peripheral arterial disease in patients with obese sarcopenia undergoing hemodialysis
}

\author{
SHUN-LI TIAN ${ }^{1}$, KAI ZHANG ${ }^{1}$ and PENG-CHENG XU ${ }^{2}$ \\ ${ }^{1}$ Department of Geratology, Tianjin Geriatric Institute; \\ ${ }^{2}$ Division of Nephrology, Tianjin Medical University General Hospital, Tianjin 300052, P.R. China
}

Received June 14, 2016; Accepted March 24, 2017

DOI: $10.3892 /$ etm.2018.6002

\begin{abstract}
Background peripheral arterial disease (PAD) is a common complication in patients undergoing dialysis, which reduces the quality of life and increases the risk of mortality. Recent literature has documented an association between increased visceral fat (VF) content and a proatherogenic factors in end-stage renal disease. The present study investigated the prevalence of PAD in patients undergoing hemodialysis. PAD was determined as an ankle-brachial index $<0.9$. Additionally, VF content was determined using multiple frequency bioelectrical impendence analysis. The nutritional status of the patients was evaluated by subjective global assessment and endothelial function was measured by ultrasonographic brachial artery flow-mediated dilatation. Patients divided into two groups (malnourished and non-malnourished) with two further subgroups in each (high VF and low VF content). The prevalence of PAD was identified to be significantly higher in patients with a high VF mass compared with a low VF mass in non-malnourished patients. PAD was significantly more common in malnourished patients compared with non-malnourished patients $(\mathrm{P}<0.01)$. The presence of PAD in patients undergoing hemodialysis was identified to be significantly correlated with age, diabetes mellitus (DM) status VF content, malnutrition, serum albumin level, diastolic blood pressure and log C-reactive protein levels. Furthermore, logistic regression analysis determined that age, DM, VF content and malnutrition were significant independent risk factors for PAD in patients undergoing hemodialysis. In conclusion, the results of the present study indicated that obesity and malnutrition act synergistically to increase the risk of PAD in patients undergoing dialysis.
\end{abstract}

Correspondence to: Dr Peng-Cheng Xu, Division of Nephrology, Tianjin Medical University General Hospital, 154 Anshan Road, Tianjin 300052, P.R. China

E-mail:nkxpc@163.com

Key words: atherosclerosis, dialysis, obesity, PAOD, nutrition

\section{Introduction}

Peripheral arterial disease (PAD) is an atherosclerotic process that manifests in the lower extremities, which is a common complication of end-stage renal disease (ESRD) $(1,2)$ and results in a reduced quality of life (3), in addition to an increased risk of mortality (4-6). While PAD is likely an important marker of the cardiovascular status, it has not been researched as thoroughly as coronary artery disease and its pathogenesis in patients undergoing dialysis is not well understood (7).

Malnutrition is frequently observed in patients with ESRD (8) and is associated with inflammation and atherosclerotic complications $(9,10)$. Malnutrition, assessed by subjective global assessment (SGA) (11), has also been associated with indirect cardiovascular risk markers, including carotid intima-mediated thickness (12) and patient mortality (13).

There has been an epidemiological rise of obesity in the general population worldwide; the incidence of obesity is also increased in those undergoing dialysis, including those with ESRD (14). Patients with ESRD and obese sarcopenia, defined as a high visceral fat (VF) content, have particularly poor outcomes (15). Furthermore, a previous study has associated visceral fat (VF) accumulation in patients with ESRD with proatherogenic hyperlipidemia and inflammation (13). However, it remains unclear how nutritional status affects the development of PAD in patients undergoing dialysis.

The present observational and cross-sectional study analyzed the presence of PAD in patients undergoing hemodialysis using ankle-brachial index (ABI) and body composition measurements.

\section{Patients and methods}

Patient selection. A total of 210 patients with ESRD undergoing hemodialysis at the Outpatient Clinic of the Division of Nephrology of Tianjin Medical University General Hospital (Tianjin, China) were analyzed during a single outpatient visit between January and October 2015. The mean age of the patients included in the present study was 60 years old, with 52\% females and $48 \%$ males. Patients undergoing hemodialysis for $<3$ months or patients unwilling or unsuitable for measurement by bioelectrical impedance analysis; for example, due to significant cognitive impairment, inability to 
walk, joint replacement or the presence of a cardiac pacemaker, were excluded. The ethical committee of Tianjin Medical University General Hospital approved the present study and written informed consent was obtained from all patients.

ABI measurement. ABI was measured as described by Feigelson et al (16). An ABI was calculated for each leg and the lowest value was used to group patients according to the presence $(\mathrm{ABI}<0.9)$ or absence $(\mathrm{ABI} \geq 0.9)$ of PAD.

SGA for malnutrition. A dedicated dietician performed a SGA for malnutrition. The SGA method included six subjective assessments, of which three were based on the patient's history (weight loss, presence of anorexia and vomiting) and three were based on the physician's grading (muscle wasting, presence of edema and loss of subcutaneous fat) (17). On the basis of these assessments, the patients' were divided into non-malnutrition or malnutrition groups, as described previously (17).

Body composition analysis. The body composition was measured by bioelectrical impendence analysis (BIA) using an MC-190 Multifrequency Body Composition Indicator (Tanita Corporation, Tokyo, Japan), with patients measured whilst standing and without shoes and socks, according to the manufacturer's protocol. This device uses multifrequency $(5,50$ and $250 \mathrm{kHz})$ BIA technology and has eight tactile electrodes, of which four are in contact with the palm and thumb of each hand, while the other four are in contact with the feet. The total fat and VF levels are determined by a proprietary equation developed by the manufacturer. Patients in the malnutrition and non-malnutrition groups were divided into high VF and low VF subgroups based upon whether their VF level was above or below the median, respectively, which was calculated separately for males and females.

Flow-mediated dilatation (FMD). Non-invasive endothelium-dependent FMD was measured for each patient after a 10 min rest period, in a supine position and with an empty stomach on a non-dialysis day. The brachial artery, without an artery-venous fistula, was visualized in a longitudinal section $15 \mathrm{~cm}$ above the antecubital fossa using a Acuson 128XP/10 Ultrasound Machine with a $7 \mathrm{MHz}$ Linear Array Transducer (Siemens AG, Munich, Germany). The baseline brachial artery diameter was measured by automated wall tracking using a Sonoline G50 Digital Ultrasound system (Siemens) as described previously (18). Furthermore, a pneumatic cuff was then inflated to $50 \mathrm{mmHg}$ above the systolic blood pressure (SBP) and kept on the forearm for 5 min to induce reactive hyperemia. The percentage change in the brachial artery diameter 1 min after cuff release compared with the baseline diameter was recorded as the FMD.

Demographic and clinical data collection. Previously diagnosed coronary artery disease (previous myocardial infarction, ischemia detected by cardiogram abnormality, a history of coronary artery bypass grafting or coronary stent implantation) or a history of cerebrovascular events was classified as cardiovascular or cerebrovascular disease (CVD). A history of smoking (current or former) was defined as a $\geq 1$ pack-year if tobacco use based on patient interviews or chart documentation.

Statistical analysis. Statistical analysis was performed using SPSS software (version 15.0; SPSS, Inc., Chicago, IL, USA). Results for continuous variables are presented as the mean \pm standard deviation and results for categorical variables are presented as percentages. The statistical significance of differences between the low and high VF groups (normal nutrition and malnourishment) were determined using a Student's t-test for comparison between two variables and one-way multivariate analysis of variance for continuous variables, while the $\chi^{2}$ test was used for categorical variables. Bivariate (Pearson) correlation analysis was conducted to determine the association between PAD and other variables. Variables that were significantly correlated with PAD were selected for binary logistic regression analysis to determine the independent risk factors for PAD. $\mathrm{P}<0.05$ (two-tailed) was considered to indicate a statistically significant difference.

\section{Results}

Clinicopathological characteristics of patients undergoing hemodialysis based upon their nutritional status and VF level. A total of 210 patients (mean age, 60 years old; 52\% female and $48 \%$ male) were available for analysis in the present study. The baseline clinicopathological characteristics are summarized in Table I. Furthermore, patients with a high VF had a significantly higher body mass index (BMI) compared with those with a low $\mathrm{VF}$, both in the malnutrition and non-malnutrition groups (both $\mathrm{P}<0.05$ ) and the BMI of malnourished patients with a low VF was significantly lower compared with that of non-malnourished patients with a

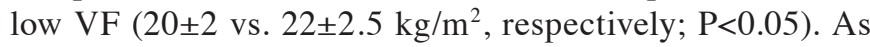
expected, the serum albumin level was significantly lower in the malnutrition group compared with the non-malnutrition groups, in patients with low VF and with high VF (both $\mathrm{P}<0.05)$. Additionally, SBP and diastolic blood pressure (DBP) was comparable between the groups, except that the DBP was significantly lower in patients with a high VF in the malnutrition group compared with the non-malnutrition group ( $69 \pm 10$ vs. $78 \pm 10$, respectively; $\mathrm{P}<0.05)$. In the present study, malnourished patients with a high VF content had worse endothelial dysfunction (by FMD) compared with patients with a low VF content. Moreover, in high VF group, malnourished patients showed decreased FMD than patients with normal nutrition.

Risk factors for PAD in patients undergoing hemodialysis. The prevalence of PAD was $28 \%$ across all patients included in the study and it was significantly higher among malnourished patients compared with non-malnourished patients (Fig. 1; $\mathrm{P}<0.01)$. However, non-malnourished patients with a high $\mathrm{VF}$ had a significantly higher prevalence of PAD compared with those with a lower VF content (29.5 vs. 12.5\%, respectively; $\mathrm{P}=0.007)$. Furthermore, bivariate correlation analysis revealed that the age, diabetic mellitus (DM), VF, malnutrition, serum albumin level, DBP and log C-reactive protein (CRP) were significantly correlated with $\mathrm{PAD}($ all $\mathrm{P}<0.05)$ and subsequent logistic regression analysis determined that age, DM, VF 
Table I. Comparison of variables between the low and high VF mass groups in well-nourished and malnourished patients undergoing hemodialysis.

Non-malnourished

Malnourished

\begin{tabular}{|c|c|c|c|c|c|}
\hline \multirow[b]{2}{*}{ Variable } & & & \multirow[b]{2}{*}{ Significance } \\
\hline & Low VF (n=136) & High VF (n=32) & Low VF $(n=20)$ & High VF (n=22) & \\
\hline Age (years) & $54 \pm 15$ & $66 \pm 10$ & $64 \pm 12$ & $72 \pm 6$ & $\mathrm{ABCD}$ \\
\hline Gender (male \%) & 56 & 50 & 23 & 41 & $\mathrm{~B}$ \\
\hline $\mathrm{DM}(\%)$ & 28 & 45 & 30 & 63 & $\mathrm{AC}$ \\
\hline CVD (\%) & 12 & 35 & 18 & 33 & A \\
\hline Smokers (\%) & 27 & 42 & 10 & 27 & NS \\
\hline Dialysis duration (m) & $13 \pm 18$ & $17 \pm 21$ & $15 \pm 18$ & $11 \pm 13$ & NS \\
\hline Height (cm) & $160 \pm 9$ & $160 \pm 8$ & $155 \pm 8$ & $159 \pm 8$ & $\mathrm{~B}$ \\
\hline Weight (kg) & $57 \pm 9$ & $67 \pm 10$ & $47 \pm 8$ & $61 \pm 10$ & $\mathrm{ABCD}$ \\
\hline BMI $\left(\mathrm{kg} / \mathrm{m}^{2}\right)$ & $22 \pm 2.5$ & $26 \pm 3$ & $20 \pm 2$ & $25 \pm 4$ & $\mathrm{ABC}$ \\
\hline Alb $(\mathrm{g} / \mathrm{l})$ & $39.9 \pm 6$ & $39.6 \pm 4$ & $36.8 \pm 4$ & $35.6 \pm 4$ & $\mathrm{BD}$ \\
\hline $\mathrm{SBP}(\mathrm{mmHg})$ & $134 \pm 20$ & $138 \pm 21$ & $133 \pm 31$ & $125 \pm 22$ & NS \\
\hline DBP (mmHg) & $80 \pm 15$ & $78 \pm 10$ & $75 \pm 13$ & $69 \pm 10$ & $\mathrm{D}$ \\
\hline TG (mmol/l) & $2.4 \pm 1.7$ & $2.8 \pm 1.8$ & $2.5 \pm 1.6$ & $2.9 \pm 2$ & NS \\
\hline $\mathrm{TC}(\mathrm{mmol} / \mathrm{l})$ & $5.1 \pm 1.1$ & $5.0 \pm 1$ & $6.1 \pm 1.5$ & $5.2 \pm 1.2$ & $\mathrm{~B}$ \\
\hline HDL (mmol/l) & $1.2 \pm 0.3$ & $1 \pm 0.2$ & $1.4 \pm 0.4$ & $1.1 \pm 0.3$ & $\mathrm{ABC}$ \\
\hline LDL (mmol/l) & $3 \pm 0.9$ & $3 \pm 1$ & $3.8 \pm 1.1$ & $3 \pm 0.8$ & $\mathrm{BC}$ \\
\hline Glu (mmol/l) & $5.4 \pm 1.7$ & $6.3 \pm 2.6$ & $6.3 \pm 2.7$ & $6.5 \pm 2$ & A \\
\hline LnCRP & $0.48 \pm 1.5$ & $1.2 \pm 1.5$ & $0.9 \pm 1.1$ & $2.1 \pm 1.4$ & $\mathrm{ACD}$ \\
\hline $\mathrm{Ca}(\mathrm{mmol} / \mathrm{l})$ & $2.3 \pm 0.4$ & $2.4 \pm 0.3$ & $2.2 \pm 0.4$ & $2.5 \pm 0.3$ & NS \\
\hline $\mathrm{P}(\mathrm{mmol} / \mathrm{l})$ & $1.6 \pm 0.4$ & $1.5 \pm 0.4$ & $1.4 \pm 0.5$ & $1.5 \pm 0.4$ & NS \\
\hline BUN (mmol/l) & $23.5 \pm 6$ & $21.5 \pm 5$ & $19.7 \pm 7.5$ & $18.6 \pm 7.3$ & $\mathrm{AB}$ \\
\hline $\operatorname{Scr}(\mu \mathrm{mol} / \mathrm{l})$ & $918 \pm 288$ & $880 \pm 277$ & $749 \pm 352$ & $792 \pm 263$ & B \\
\hline KT/V (total) & $1.8 \pm 0.5$ & $1.8 \pm 0.6$ & $1.9 \pm 0.4$ & $1.8 \pm 1.8$ & NS \\
\hline KT/V (renal) & $0.5 \pm 0.6$ & $0.4 \pm 0.7$ & $0.4 \pm 0.4$ & $0.3 \pm 0.3$ & NS \\
\hline Fat (kg) & $14 \pm 5$ & $23 \pm 8$ & $11 \pm 3$ & $21 \pm 8$ & $\mathrm{AC}$ \\
\hline $\mathrm{Hb}(\mathrm{g} / \mathrm{l})$ & $115 \pm 17$ & $118 \pm 15$ & $109 \pm 21$ & $115 \pm 20$ & NS \\
\hline Total fat (\%) & $24 \pm 8$ & $33 \pm 10$ & $24 \pm 7$ & $31 \pm 14$ & $\mathrm{AC}$ \\
\hline FMD (\%) & $8.6 \pm 5.5$ & $7.3 \pm 3.6$ & $8.6 \pm 4.5$ & $4.6 \pm 2.4$ & $\mathrm{CD}$ \\
\hline VF & $7.2 \pm 3.3$ & $12.8 \pm 3.5$ & $5.6 \pm 2.2$ & $12.5 \pm 3$ & $\mathrm{ABC}$ \\
\hline
\end{tabular}

A and C: Significant difference between low and high VF groups within the malnutrition or non-malnutrition groups. B and D: Significant difference between the malnutrition and non-malnutrition groups within low and high VF groups. NS, no significant difference between each group. DM, diabetic mellitus; CVD, cardiovascular or cerebrovascular disease; BMI, body mass index; Alb, serum albumin; SBP, systolic blood pressure; DBP, diastolic blood pressure; TG, triglyceride; TC, total cholesterol; HDL, high-density lipoprotein; LDL, low-density lipoprotein; LnCRP, $\log$ C-reactive protein; BUN, blood urea nitrogen; Scr, serum creatinine; KT/V, clearance of urine; Hb, hemoglobin; FMD, flow-mediated dilatation; VF, visceral fat.

content and malnutrition were independent risk factors for PAD (all P<0.05) (Table II).

\section{Discussion}

The present study revealed an association between nutritional status and VF content and the prevalence of PAD in patients undergoing hemodialysis. Malnourished patients with a high VF mass (obese sarcopenia) had the highest prevalence of PAD in the hemodialysis population of the present study. This is similar to the results of a previous study (15), which identified the detrimental effects of malnutrition in the presence of obesity by demonstrating that obese sarcopenia was associated with inflammation and increased mortality in ESRD patients.

Notably, increased VF was identified to be an independent risk factor for PAD in the present study. Although no previous studies, to the best of our knowledge, have explored the association between VF and PAD in patients with ESRD, it has been revealed that visceral obesity is associated with uremic dyslipidemia, inflammation and arterial stiffness (19), in addition to cardiovascular mortality (20) in patients with ESRD.

The underlying molecular mechanisms of the association between adiposity and CVD are not fully understood (21); however, it is well known that adipose tissue, in addition to 
Table II. Univariate and multivariate analysis of selected variables and peripheral arterial disease patients undergoing hemodialysis.

\begin{tabular}{lrrlll}
\hline & \multicolumn{4}{c}{ Analysis type } \\
\cline { 2 - 3 } & \multicolumn{2}{c}{ Univariate } & & \multicolumn{2}{c}{ Multivariate } \\
\cline { 2 - 3 } \cline { 5 - 6 } Variable & R-value & P-value & & $\operatorname{Exp}(\beta)$ & P-value \\
\hline Age & 0.304 & $<0.010$ & & 1.036 & 0.047 \\
DM & 0.242 & $<0.010$ & & 2.11 & 0.045 \\
Visceral fat & 0.211 & $<0.010$ & & 1.11 & 0.030 \\
Malnutrition & 0.277 & $<0.010$ & & 4.53 & 0.010 \\
Alb & -0.170 & 0.014 & & \\
LnCRP & 0.168 & 0.016 & & \\
DBP & -0.226 & $<0.010$ & & \\
\cline { 3 - 4 } & & &
\end{tabular}

Univariate analysis was bivariate correlation analysis and multivariate analysis was logistic regression analysis. DM, diabetic mellitus; Alb, serum albumin; LnCRP, $\log$ C-reactive protein; DBP, diastolic blood pressure; $\operatorname{Exp}(\beta)$, odds ratio for each variable in multivariate regression.

storing energy in the form of triglycerides, is a highly active endocrine tissue that secretes a large variety of cytokines, several of which are thought to be associated with cardiovascular disease (13). Furthermore, systemic inflammation is considered to be an important mediator of CVD in patients with ESRD, while VF has been identified to be positively correlated with low-grade inflammation in this patient group (22). In the present study, patients with a higher VF mass had significantly higher CRP levels, particularly if they were also malnourished. Similarly, levels of antiatherogenic high-density lipoprotein q43 known to be low in obese patients with ESRD (22) and the present study identified that patients with increased abdominal fat had a more atherogenic lipid profile.

A risk factor for PAD is endothelial dysfunction, which has previously been determined to be associated with obesity in non-uremic individuals (13). In the present study, malnourished patients with a high VF content had worse endothelial dysfunction. This confirms earlier reports of an association between fat content and FMD (13), in addition to between FMD and the adipokines, adiponectin, leptin and visfatin, in patients undergoing dialysis (13). The present study did not show an association between FMD and PAD prevalence, which is contrary to what previous studies have identified. However, the patients undergoing hemodialysis in the present study had several risk factors for PAD, including hypertension, dyslipidemia, malnutrition, obesity, diabetes, smoking, CRP level and CVD complications, which could contribute to a lower FMD independently of PAD (23).

The present study had a number of limitations, including that a causal relationship between PAD and obese sarcopenia could not be determined by a cross-sectional observation study and the estimation of the body composition of patients undergoing hemodialysis by BIA may not be as accurate as that of general subjects due to volume overload. In addition,

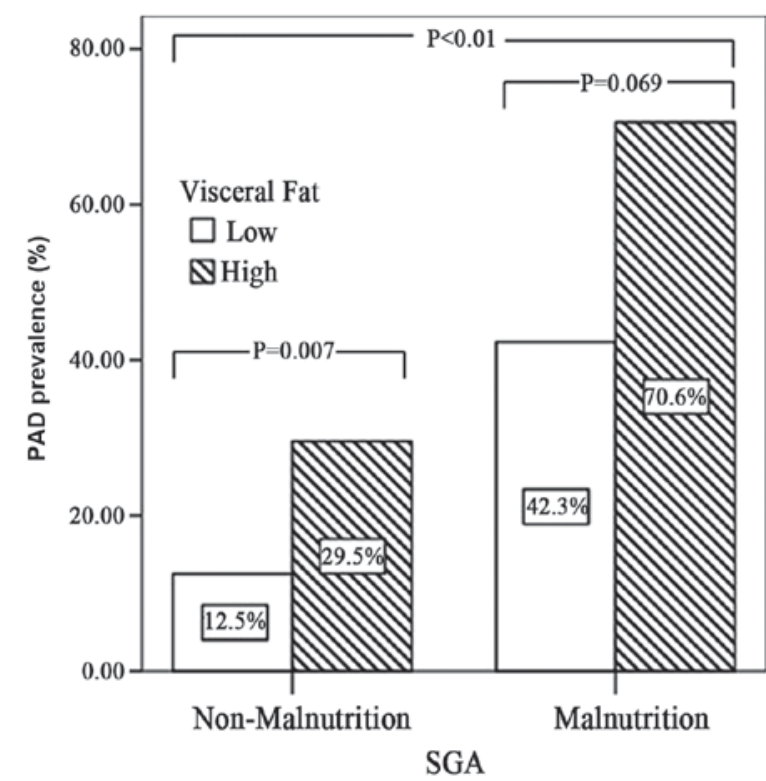

Figure 1. Prevalence of PAD in non-malnourished and malnourished patients with a low VF or high VF content undergoing hemodialysis. PAD, peripheral arterial disease; VF, visceral fat; SGA, subjective global assessment.

the levels of atherogenetic adipokines, including leptin and adiponectin, were not tested in the present study.

In conclusion, obesity and malnutrition were demonstrated to have a synergistic effect on increasing the risk of PAD in patients undergoing hemodialysis. The association between a high VF content and increased risk of PAD has attracted the attention of nephrologists for cardiovascular disease detection and prevention, particularly due to the increasing prevalence of obesity in patients with ESRD (14). The present study presents an association between PAD and obese sarcopenia, which implies that some intervention is required to decrease the incidence of cardiovascular complications in patients with ESRD. Further studies are required to clarify whether improving nutritional status and decreasing visceral fat-mass may help to reduce cardiovascular complications in patients with ESRD.

\section{Acknowledgements}

Not applicable.

\section{Funding}

The present study was funded by the Tianjin Natural Science Foundation Youth Project (grant no. 15JCQNJC12500).

\section{Availability of data and materials}

The datasets used and/or analyzed during the current study are available from the corresponding author on reasonable request.

\section{Authors' contributions}

ST contributed to the study design and data analysis. KZ collected and analyzed data. PX contributed to the design and 
drafting of the manuscript, agrees to be accountable for all aspects of the work and gave final approval of the version to be published.

\section{Ethics approval and consent to participate}

The ethics committee of Tianjin Medical University General Hospital approved the present study and written informed consent was obtained from all patients.

\section{Consent for publication}

All patients provided written informed consent for publication.

\section{Competing interests}

The authors declare that they have no competing interests.

\section{References}

1. Liu JH, Lin HH, Yang YF, Liu YL, Kuo HL, Wang IK, Chou CY and Huang CC: Subclinical peripheral artery disease in patients undergoing peritoneal dialysis: Risk factors and outcome. Perit Dial Int 29: 64-71, 2009.

2. Chen SC, Su HM, Chang JM, Liu WC, Tsai JC, Tsai YC, Lin MY, Hwang SJ and Chen HC: Increasing prevalence of peripheral artery occlusive disease in hemodialysis patients: A 2-year follow-up. Am J Med Sci 343: 440-445, 2012.

3. Tian SL, Tian XK, Han QF, Axelsson J and Wang T: Presence of peripheral arterial disease predicts loss of residual renal function in incident CAPD patients. Perit Dial Int 32: 67-72, 2012.

4. Jimenez ZN, Pereira BJ, Romão JE Jr, Makida SC, Abensur H, Moyses RM and Elias RM: Ankle-brachial index: A simple way to predict mortality among patients on hemodialysis-a prospective study. PLoS One 7: e42290, 2012.

5. Otsubo S, Kitamura M, Wakaume T, Yajima A, Ishihara M, Takasaki M, Ueda S, Sugimoto H, Otsubo K, Kimata N, et al: Association of peripheral artery disease and long-term mortality in hemodialysis patients. Int Urol Nephrol 44: 569-573, 2012.

6. Tian SL, Tian XK, Han QF and Wang T: Peripheral arterial disease predicts overall and cardiovascular mortality in peritoneal dialysis patients. Ren Fail 34: 1010-1014, 2012.

7. Matsuzawa R, Aoyama N and Yoshida A: Clinical characteristics of patients on hemodialysis with peripheral arterial disease. Angiology 66: 911-917, 2015 .

8. Locatelli F, Fouque D, HeimburgerO, Drüeke TB, Cannata-Andía JB, Hörl WH and Ritz E: Nutritional status in dialysis patients: A European consensus. Nephrol Dial Transplant 17: 563-572, 2002.
9. Papagianni A, Kokolina E, Kalovoulos M, Vainas A, Dimitriadis C and Memmos D: Carotid atherosclerosis is associated with inflammation, malnutrition and intercellular adhesion molecule-1 in patients on continuous ambulatory peritoneal dialysis. Nephrol Dial Transplant 19: 1258-1263, 2004.

10. Oka RK and Alley HF: Differences in nutrition status by body mass index in patients with peripheral artery disease. J Vasc Nurs 30: 77-87, 2012.

11. Pecoits-Filho R, Lindholm B and Stenvinkel P: The malnutrition, inflammation, and atherosclerosis (MIA) syndrome-the heart of the matter. Nephrol Dial Transplant 17 (Suppl 11): S28-S31, 2002.

12. Choi HY, Lee JE, Han SH, Yoo TH, Kim BS, Park HC, Kang SW, Choi KH, Ha SK, Lee HY and Han DS: Association of inflammation and protein-energy wasting with endothelial dysfunction in peritoneal dialysis patients. Nephrol Dial Tranplant 25: 1266-1271, 2010.

13. Zoccali C: The obesity epidemics in ESRD: From wasting to waist? Nephrol Dial Transplant 24: 376-380, 2009.

14. Honda H, Qureshi AR, Axelsson J, Heimburger O, Suliman ME, Barany P, Stenvinkel P and Lindholm B: Obese sarcopenia in patients with end-stage renal disease is associated with inflammation and increased mortality. Am J Clin Nutr 86: 633-638, 2007.

15. Feigelson HS, Criqui MH, Fronek A, Langer RD and Molgaard CA: Screening for peripheral arterial disease: The sensitivity, specificity, and predictive value of noninvasive tests in a defined population. Am J Epidemiol 140: 526-534, 1994.

16. Young GA, Kopple JD, Lindholm B, Vonesh EF, De Vecchi A, Scalamogna A, Castelnova C, Oreopoulos DG, Anderson GH, Bergstrom J, et al: Nutritional assessment of continuous ambulatory peritoneal dialysis patients: An international study. Am J Kidney Dis 17: 462-471, 1991.

17. Lee JW, Lee HR, Shim JY, Im JA, Kim SH, Choi H and Lee DC: Viscerally obese women with normal body weight have greater brachial-ankle pulse wave velocity than nonviscerally obese women with excessive body weight. Clin Endocrinol (Oxf) 66: 572-578, 2007.

18. Postorino M, Marino C, Tripepi G and Zoccali C; CREDIT (Calabria Registry of Dialysis and Transplantation) Working Group: Abdominal obesity and all-cause and cardiovascular mortality in end-stage renal disease. J Am Coll Cardiol 53: 1265-1272, 2009.

19. Fantuzzi G and Mazzone T: Adipose tissue and atherosclerosis: Exploring the connection. Arterioscler Thromb Vasc Biol 27: 996-1003, 2007.

20. Mafra D, Guebre-Egziabher F and Fouque D: Body mass index, muscle and fat in chronic kidney disease: Questions about survival. Nephrol Dial Transplant 23: 2461-2466, 2008.

21. Berg AH and Scherer PE: Adipose tissue, inflammation, and cardiovascular disease. Circ Res 96: 939-949, 2005.

22. Axelsson J, Rashid Qureshi A, Suliman ME, Honda H, Pecoits-Filho R, Heimbürger O, Lindholm B, Cederholm T and Stenvinkel P: Truncal fat mass as a contributor to inflammation in end-stage renal disease. Am J Clin Nutr 80: 1222-1229, 2004.

23. Kuang L and Wong ND: Abstract 38: HDL cholesterol, inflammation and peripheral arterial disease in U.S. adults. Circulation 129 (Suppl 1): A38, 2014. 